\title{
Stimulation of Plasticity and Functional Recovery After Stroke - Cell-based and Pharmacological Therapy
}

\author{
Michael Chopp ${ }^{1}$ and $\mathrm{Yi} \mathrm{Li}^{2}$ \\ 1. Scientific Director, Neuroscience Research, Department of Neurology, Henry Ford Hospital and Department of Physics, Oakland University, \\ 2. Neurologist, Department of Neurology, Henry Ford Hospital
}

\section{Abstract}

Stroke is a major cause of mortality and morbidity worldwide. There are two approaches to treat stroke, a neuroprotective approach that focuses on reducing the lesion and a neurorestorative one, designed to promote neurological recovery by treating the entire central nervous system (CNS). To date, clinical trials of neuroprotective agents for stroke have failed. Recently, cell-based and pharmacological neurorestorative therapies have shown enormous promise in reducing neurological deficits caused by stroke. This article will briefly describe preclinical studies on how the injured CNS can be stimulated by cell-based and pharmacological therapies to amplify its intrinsic restorative processes and thereby lead to improvement in neurological function post-stroke.
\end{abstract}

\section{Keywords}

Stroke, cell therapy, pharmacological therapy, stimulation of plasticity, functional recovery, neurorestoration

Disclosure: The authors have no conflicts of interest to declare.

Acknowledgements: This work was supported by R01 AG037506, R01 NS066041 and P01 NS023393.

Received: 5 May 2011 Accepted: 23 May 2011 Citation: European Neurological Review, 2011;6(2):97-100 DOl:10.17925/ENR.2011.06.02.97

Correspondence: Michael Chopp, Neurology Research, E\&R Bldg., Room 3056, Henry Ford Hospital, 2799 West Grand Boulevard, Detroit, MI 48202, US.

E: chopp@neuro.hfh.edu

Support: The publication of this article was funded by EVER Neuro Pharma GmbH. The views and opinions expressed are those of the authors and not necessarily those of EVER Neuro Pharma GmbH.

For decades, the primary approach and goal of therapy for stroke and neural injury have been the treatment of the injured tissue, with intervention designed to reduce the volume of cerebral infarction. Enormous effort has gone into the development of neuroprotective agents, including free radical scavengers, ${ }^{1-3}$ glutamate antagonists, ${ }^{4,5}$ among a myriad of others..$^{6-8}$ Neuroprotective agents developed in the laboratory were translated into clinical trials and all have failed. ${ }^{9.10}$ Reasons for these failures are multifaceted and in part may be attributed to inadequate, and often unrealistic, treatment protocols associated with dosing and time of administration, naively translated from preclinical studies to the clinic. The only agent that has been developed and is now in clinical use for the treatment of stroke is recombinant tissue plasminogen activator (rtPA) employed for thrombolysis. ${ }^{11,12}$ rtPA is, however, not a neuroprotective agent, but, essentially an agent that improves tissue perfusion by lysing the offending clot. The therapeutic window for rtPA, however, is merely 4.5 hours, $^{13}$ and until recently only three hours. ${ }^{14,15}$ However, currently in the US, fewer than $5 \%$ of patients receive rtPA, with the primary reason being the relatively short therapeutic window. ${ }^{16}$ In addition, rtPA has a serious adverse side effect of increasing the rate of haemorrhagic transformation. ${ }^{17}$ rtPA also cannot be employed for haemorrhagic stroke. Therefore, there is a compelling need to develop therapeutic agents whose use extends well beyond the first few hours of stroke and can be employed to treat all stroke patients. These agents would be designed for treatment days and weeks after stroke. To do this requires a paradigm shift away from neuroprotective agents for stroke that treat the ischaemic lesion, to that of neurorestorative agents which treat the intact or compromised cerebral tissue, to promote brain plasticity and thereby remodel the brain to compensate for the damaged tissue. In this article, we will review select ways to stimulate brain plasticity post-stroke and thereby improve functional outcome.

The majority of stroke patients, particularly younger patients, show improvement in neurological function over time. This improvement may be associated with compensatory processes, which may be attributed to the induction of brain plasticity. ${ }^{18}$ Post-stroke or brain injury, cerebral tissue is primed for recovery. The injured and affected cerebral tissue, in various ways reverts to a quasi-ontogenous or developmental state, expressing genes and proteins that are developmental and that lead to brain remodeling. ${ }^{19}$ In this quasi-developmental state, angiogenesis, neurogenesis and synaptogenesis are evident. These restorative processes that are interdependent essentially remodel the brain and lead to improved neurological function. However, these restorative processes are often inadequate to fully restore neurological function and many stroke patients are left with severe neurological deficits. The essential question therefore is, whether we can amplify these restorative processes so that neurological function can be enhanced post-stroke. In this article, we will briefly describe ways to do this. Cell-based and pharmacological therapies can stimulate essential 
restorative processes and thereby lead to improvement in neurological function. We will describe preclinical work and review the applications of these cell-based and pharmacological restorative approaches to the stroke patient.

\section{Cell-based Therapies for Stroke}

Cells are essentially a living polypharmacy, providing multiple restorative factors that are biologically titrated to the needs of the tissue. Although stem cells have the capacity to differentiate into all cells, ${ }^{20,21}$ their use, thus far for the treatment of stroke, is not connected with this differentiation. Exogenously administered cells appear to stimulate endogenous restorative processes and do not replace injured cerebral tissue..$^{22-24}$ The administered cells release many trophic factors and more importantly stimulate parenchymal cells, primarily astrocytes, to produce proteins that amplify brain plasticity. ${ }^{25,26} \mathrm{We}$, and others, have shown that exogenously administered cells delivered by a vascular route greatly amplify the generation of neuroblasts in the subventricular zone. ${ }^{27}$ These neuroblasts migrate to the site of injury and interact with cerebral vasculature, particularly angiogenic vessels. ${ }^{28,29}$ Concurrently, angiogenesis is upregulated, primarily in or near to the boundary zone of the ischemic lesion..$^{30,31}$ These angiogenic vessels produce an array of factors, including brain-derived neurotrophic factor (BDNF) and vascular endothelial growth factor (VEGF), which promote neurogenesis. ${ }^{32,33}$ Therefore, angiogenesis and neurogenesis are coupled restorative systems. The angiogenic vessels promote differentiation of the neuroblasts and the neuroblasts promote further angiogenesis and upregulate the expression of agents, such as angiopoietin 1, which leads to the maturation of the newly formed vasculature. ${ }^{34}$ Concurrent and likely driven by these processes, is an extensive amplification of neurite outgrowth and increased axonal density. ${ }^{3,36}$ These rewiring events are present in the ipsilateral hemisphere, ${ }^{37,38}$ as well as in the contralateral hemisphere and there is a robust and highly significant correlation between neurite outgrowth and functional recovery, particularly in the contralateral hemisphere. ${ }^{3,40}$ Of substantial interest, it should be noted that not only is there bilateral cerebral hemisphere response and plasticity to cell therapy, but, also there is neurite outgrowth in the spinal cord, with axonal extension from the intact to the denervated cord and enhanced plasticity of the cortical spinal tract, which robustly correlate with improvement of neurological function post-stroke. ${ }^{41}$ Thus, cell therapies amplify endogenous restorative events that facilitate neurological recovery. There have been many studies using a large variety of cells for the treatment of experimental stroke, ranging from adult mesenchymal cells, ${ }^{18,42,43}$ to cord blood, ${ }^{44}$ cord tissue $^{45}$ and an array of progenitor and stem cells, ${ }^{46,47}$ including embryonic stem cells. ${ }^{48}$

The transplantation of stem/progenitor cells is a key element in the rapidly growing field of regenerative medicine. Based on their ability to rescue and/or repair injured tissue and partially restore organ function, multiple types of stem/progenitor cells have already entered into clinical trials. Safety for various cells has been demonstrated in stroke patients. Several institutions have carried out Phase I clinical trials with intravenous autologous bone marrow transplantation for stroke patients and have reported preliminary results. ${ }^{49-52}$

\section{Pharmacological Therapy for Recovery of Function}

Cells are not the only means by which to stimulate recovery via the induction of brain plasticity. There are a number of pharmacological agents, some of which mimic or reflect developmental processes, which promote brain recovery. Trophic factors, such as BDNF, ${ }^{32,53}$ hepatocyte growth factor (HGF) $)^{54,55}$ and granulocyte-macrophage colony-stimulating factor (GM-CSF) $)^{56}$, and other agents such as minocycline ${ }^{57}$ have been demonstrated to provide restorative therapeutic benefit in preclinical studies and have moved into clinical trials..$^{58,59}$

Our laboratory has pioneered the use of phosphodiestrase 5 inhibitors, ${ }^{60-63}$ statins, $^{64-66}$ and agents that increase high-density lipoproteins and hormones such as thymosin beta $4{ }^{67}$ erythropoietin ${ }^{68,69}$ and carbamylated erythropoietin for the treatment of stroke ${ }^{70}$ and neural injury, ${ }^{71}$ and we have also employed the multifactor restorative agent cerebrolysin for stroke therapy. ${ }^{72,73}$

All the pharmacological agents tested so far, which show evidence of being neurorestorative, induce brain plasticity, angiogenesis, neurogenesis and synaptogenesis. The molecular and signal transduction pathways by which these agents promote brain plasticity have also been investigated, and they appear to activate specific signaling pathways, such as PI3K/Akt ${ }^{72,74-76}$ and sonic hedgehog (Shh). ${ }^{77,78}$ This article, however, is not a forum to discuss these pathways. We focus here on the observation that pharmacological agents by various means and multiple signal transduction pathways can induce CNS plasticity that enhances functional recovery from stroke and neural injury.

The translation of these restorative agents from the laboratory to the clinic has to be performed with care and laboratory testing must be performed to simulate as close as possible clinical conditions. Not doing so can result in failure of a trial and poor outcome for stroke patients. The use of erythropoietin (EPO) to treat stroke provides an example of the poor translation of laboratory studies to the clinic leading to a negative clinical trial. ${ }^{79}$ EPO was demonstrated in multiple preclinical studies to provide potent therapeutic benefit for the treatment of stroke, and appeared to be a strong candidate for translation into the clinic. The Phase III clinical trial that was performed, however, was unsuccessful, and had to be terminated because of high mortality and adverse effects. ${ }^{79}$ Careful comparison of the clinical trial with the preclinical data illustrates the inadequate performance of preclinical studies and the failure to properly test, i.e. to perform studies in the animals that will mimic the use of the agent in the human. Of the stroke patients in the reported clinical trial, $63.4 \%$ were administered rtPA, yet prior to the performance of the clinical trial, EPO was not tested in the laboratory in conjunction with rtPA. A subsequent study from our laboratory with the combination of rtPA and EPO clearly demonstrated in animals the adverse effects observed in the human trial. ${ }^{80}$ There were other flaws associated with the EPO clinical trial, including enrollment of an unacceptably high proportion of patients that did not comply with the inclusion criteria specified in the treatment protocol. Therefore, because of inadequate preclinical study and poor recruitment, a potentially important and promising drug for the treatment of stroke has been removed from testing.

Phosphodiestrase 5 (PDE5) inhibitors are examples of an agent that shows promising therapeutic restorative effects, when administered 24 or more hours after stroke. The studies with PDE5 inhibitors arose because of early studies showing that agents that increase nitric oxide (NO), donors of NO, such as diethylenetriamine NONOate (DATA-NONOate), provide restorative therapeutic effect poststroke. ${ }^{81}$ Further studies demonstrated that the restorative effect to increasing NO could be attributed to the increase of cyclic guanosine 
monophosphate (cGMP), ${ }^{60}$ a major signalling molecule. To confirm the role of CGMP as the target for the restorative therapeutic effect of NO, we sought another way to increase CGMP without increasing NO. CGMP can be increased in tissue by inhibiting its hydrolysis via PDE5. ${ }^{63}$ Therefore, by using a PDE5 inhibitor, we would increase CGMP. ${ }^{82}$ A widely used PDE5 inhibitor is sildenafil and we have tested sildenafil in preclinical stroke studies, demonstrating its efficacy in young and old animals, ${ }^{83}$ with a therapeutic window of at least 30 days post-stroke..$^{84}$ Our studies have led to a Phase II clinical trial sponsored by Pfizer in which a PDE5 inhibitor is used as a restorative agent to treat stroke.

Cerebrolysin, a peptide-based drug, is a prime candidate for the treatment of stroke and neural injuries. ${ }^{73}$ Multiple laboratories have demonstrated the safety and efficacy of this agent in the treatment of experimental stroke. ${ }^{72,85}$ Cerebrolysin is presently in clinical trials and is in common use in many countries for the clinical treatment of stroke. ${ }^{86}$ We have demonstrated that cerebrolysin induces neurogenesis and angiogenesis in animal models of stroke and concomitantly enhances brain plasticity and recovery from stroke. ${ }^{72}$ The full potential of this drug as a restorative agent for the treatment of stroke and neural injury awaits further investigation.

In this article, we have briefly described a novel approach to the treatment of stroke and neural injury, the use of agents to stimulate endogenous recovery mechanisms. Although neuroprotection remains a vital clinical target, it is our belief that therapeutic efficacy would be maximised with a focus on neurorestoration, the amplification of intrinsic CNS processes using cell-based or pharmacological based therapies. The therapeutic approach should be to minimise damage, via neuroprotective agents, and to maximise recovery through neurorestorative agents, treating the intact brain.
Giroux C, Scatton B, Ischemic stroke: Treatment on the horizon, Eur Neurol, 1996;36:61-4.

2. Tanaka K, Fukuuchi $Y$, Nogawa S, et al., [targets of treatment in the acute phase of cerebral infarction], Rinsho Shinkeigaku, 2001:41:1052-4.

3. Nakase T, Yoshioka S, Suzuki A, Free radical scavenger, edaravone, reduces the lesion size of lacunar infarction in human brain ischemic stroke, BMC Neurol, 2011;11:39.

4. Bullock R, Strategies for neuroprotection with glutamate antagonists, Extrapolating from evidence taken from the firs stroke and head injury studies, Annals of the New York Academy of Sciences, 1995;765:272-8; discussion 298.

5. Muir KW, Glutamate-based therapeutic approaches: Clinical trials with nmda antagonists, Curr Opin Pharmacol, 2006:6:53-60.

6. Delgado P, Sahuquillo J, Poca MA, Alvarez-Sabin J, Neuroprotection in malignant mca infarction, Cerebrovasc Dis, 2006;21(Suppl. 2):99-105.

7. Perez de la Ossa N, Davalos A, Neuroprotection in cerebral infarction: The opportunity of new studies, Cerebrovasc Dis, 2007;24 (Suppl. 1):153-6.

8. Mracek J, [Past, present and future of neuroprotection], Cas Lek Cesk, 2010;149:586-90.

9. Green AR, Why do neuroprotective drugs that are so promising in animals fail in the clinic? An industry perspective, Clin Exp Pharmacol Physiol, 2002:29:1030-4.

10. Curry SH, Why have so many drugs with stellar results in laboratory stroke models failed in clinical trials? A theory based on allometric relationships, Ann NY Acad Sci, 2003;993:69-74; discussion 79-81.

11. Demaerschalk BM, Hwang HM, Leung $G$, Cost analysis review of stroke centers, telestroke, and rt-pa, Am I Manag Care, 2010;16:537-44

12. Fugate JE, Giraldo EA, Rabinstein AA, Thrombolysis for cerebral ischemia, Front Neurol, 2010;1:139.

13. Otwell JL, Phillippe HM, Dixon KS, Efficacy and safety of i.V. Alteplase therapy up to 4.5 hours after acute ischemic stroke onset, Am J Health Syst Pharm, 2010;67:1070-4.

14. Ifejika-Jones NL, Harun N, Mohammed-Rajput NA, et al., Thrombolysis with intravenous tissue plasminogen activator predicts a favorable discharge disposition in patients with acute ischemic stroke, Stroke, 2011;42:700-4.

15. Benmira S, Banda ZK, Bhattacharya V, The start of a new era for stroke treatment: Mechanical thrombectomy devices, Curr Neurovasc Res, 2011:8:75-85.

16. Fang MC, Cutler DM, Rosen $A B$, Trends in thrombolytic use for ischemic stroke in the united states, J Hosp Med, 2010;5:406-9.

17. Fagan SC, Nagaraja TN, Fenstermacher JD, et al., Hemorrhagic transformation is related to the duration of occlusion and treatment with tissue plasminogen activator in a nonembolic stroke model, Neurol Res, 2003;25:377-82.

18. Chopp M, Li Y, Zhang J, Plasticity and remodeling of brain, I Neurol Sci, 2008;265:97-101.

19. Harvey RL, Chopp M, The therapeutic effects of cellular therapy for functional recovery after brain injury, Phys Med Rehabil Clin N Am, 2003;14:S143-51.

20. McKay RD, Stem cell biology and neurodegenerative disease, Philos Trans R Soc Lond B Biol Sci, 2004;359:851-6.

21. Ross $\mathrm{JJ}$, Verfaillie CM, Evaluation of neural plasticity in adult stem cells, Philos Trans R Soc Lond B Biol Sci, 2008;363:199-205.

22. Chopp M, Li Y, Treatment of neural injury with marrow stromal cells, Lancet Neurol, 2002;1:92-100.

23. Chopp M, Li Y, Chen J, et al., Brain repair and recovery from stroke, European Neurology, 2008;3:2-5.

24. Chopp M, Li Y, Zhang ZG, Mechanisms underlying improved recovery of neurological function after stroke in the rodent after treatment with neurorestorative cell-based therapies, Stroke, 2009;40:S143-5.

25. Gao Q, Li Y, Chopp M, Bone marrow stromal cells increase astrocyte survival via upregulation of phosphoinositide 3kinase/threonine protein kinase and mitogen-activated protein kinase kinase/extracellular signal-regulated kinase pathways and stimulate astrocyte trophic factor gene expression after anaerobic insult, Neuroscience, 2005;136:123-34.

26. Li Y, Chopp M, Marrow stromal cell transplantation in stroke and traumatic brain injury, Neuroscience letters, 2009;456:120-3.

27. Shen LH, Li Y, Chopp M, Astrocytic endogenous glial cell derived neurotrophic factor production is enhanced by bone marrow stromal cell transplantation in the ischemic boundary zone after stroke in adult rats, Glia, 2010;58:1074-81.

28. Liu XS, Chopp M, Zhang XG, et al., Gene profiles and electrophysiology of doublecortin-expressing cells in the subventricular zone after ischemic stroke, $J$ Cereb subventricular zone after ische

29. Kojima T, Hirota Y, Ema M, et al., Subventricular zone-derived neural progenitor cells migrate along a blood vessel scaffold toward the post-stroke striatum, Stem cells (Dayton, Ohio), 2010;28:545-54.

30. Chopp M, Zhang ZG, Jiang Q, Neurogenesis, angiogenesis, and mri indices of functional recovery from stroke, Stroke, 2007;38:827-31

31. Onda T, Honmou O, Harada K, et al., Therapeutic benefits by human mesenchymal stem cells (hmscs) and ang-1 gene-modified hmscs after cerebral ischemia, I Cereb Blood Flow Metab, 2008:28:329-40.

32. Chen J, Zhang $\mathrm{C}$, Jiang $\mathrm{H}$, et al., Atorvastatin induction of vegf and bdnf promotes brain plasticity after stroke in mice J Cereb Blood F Metab, 2005;25:281-90.

33. Madri JA, Modeling the neurovascular niche: Implications for recovery from cns injury, J Physiol Pharmacol, 2009;60 (Suppl. 4):95-104

34. Chen J, Cui X, Zacharek A, Chopp M, Increasing ang1/tie2 expression by simvastatin treatment induces vascular stabilization and neuroblast migration after stroke, I Cell Mol Med, 2008:13(7):1348-57.

35. Shen $L H$, Xin $H$, Li Y , et al., Endogenous tissue plasminogen activator mediates bone marrow stromal cell-induced neurite remodeling after stroke in mice, Stroke, 2011;42:459-64.

36. Xin $H$, Li Y , Shen LH, et al., Increasing tpa activity in astrocytes induced by multipotent mesenchymal stromal cells facilitate neurite outgrowth after stroke in the mouse, PLOS One, 2010;5:e9027.

37. Liu Z, Li Y, Qu R, et al., Axonal sprouting into the denervated spinal cord and synaptic and postsynaptic protein expression in the spinal cord after transplantation of bone marrow stromal cell in stroke rats, Brain Research. 2007:1149:172-80.

38. Liu Z, Li Y, Zhang ZG, et al., Bone marrow stromal cells enhance inter- and intracortical axonal connections after ischemic stroke in adult rats, $J$ Cereb Blood F Metab, 2010;30:1288-95

39. Liu Z, Li Y, Zhang $X$, et al., Contralesional axonal remodeling of the corticospinal system in adult rats after stroke and bone marrow stromal cell treatment, Stroke, 2008;39:2571-7.

40. Liu Z, Li Y, Zhang RL, Cui Y, et al., Bone marrow stromal cells promote skilled motor recovery and enhance contralesional axonal connections after ischemic stroke in adult mice, stroke, 2011;42:740-4.

41. Liu Z, Zhang RL, Li Y, et al., Remodeling of the corticospinal innervation and spontaneous behavioral recovery after ischemic stroke in adult mice, Stroke, 2009;40:2546-51.

42. Miller RH, Bai L, Lennon DP, Caplan Al, The potential of mesenchymal stem cells for neural repair, Discov Med, 2010;9:236-42.

43. Vemuri MC, Chase LG, Rao MS, Mesenchymal stem cell assays and applications, Methods Mol Biol, 2011:698:3-8.

44. Chen J, Sanberg PR, Li Y, et al., Intravenous administration of human umbilical cord blood reduces behavioral deficits after stroke in rats, Stroke, 2001;32:2682-8.
45. Zhang L, Li Y, Zhang $C$, et al., Delayed administration of human umbilical tissue-derived cells improved neurological functional recovery in a rodent model of focal ischemia, Stroke, 2011:42(5):1437-44.

46. Urbaniak Hunter K, Yarbrough C, Ciacci J, Stem cells in the treatment of stroke, Adv Exp Med Biol, 2010;671:105-16

47. Shimada IS, Spees JL, Stem and progenitor cells for neurological repair: Minor issues, major hurdles, and exciting opportunities for paracrine-based therapeutics, J Cell Biochem, 2011;112:374-80.

48. Joannides AJ, Chandran S, Human embryonic stem cells: An experimental and therapeutic resource for neurological disease, J Neurol Sci, 2008;265:84-8.

49. Bang OY, Lee JS, Lee PH, Lee G, Autologous mesenchyma stem cell transplantation in stroke patients, Ann Neurol, 2005;57:874-82.

50. Suarez-Monteagudo C, Hernandez-Ramirez P, AlvarezGonzalez L, et al., Autologous bone marrow stem cell neurotransplantation in stroke patients. An open study, Restor Neurol Neurosci, 2009;27:151-61.

51. Lee JS, Hong JM, Moon GJ, et al., A long-term follow-up study of intravenous autologous mesenchymal stem cell transplantation in patients with ischemic stroke, Stem cells (Dayton, Ohio), 2010:28:1099-106.

52. Honmou $\mathrm{O}$, Houkin $\mathrm{K}$, Matsunaga $\mathrm{T}$, et al., Intravenous administration of auto serum-expanded autologous mesenchymal stem cells in stroke, Brain 2011;134(Pt 6):1790-807.

53. Pearson-Fuhrhop KM, Cramer SC, Genetic influences on neural plasticity, PM R, 2010;2:S227-40.

54. Doeppner TR, Kaltwasser B, Elali A, et al., Acute hepatocyte growth factor treatment induces long-term neuroprotection and stroke recovery via mechanisms involving neural precursor cell proliferation and differentiation, I Cereb Blood F Metab, 2010;31(5):1251-6.

55. Shang J, Deguchi $K$, Ohta $Y$, et al., Strong neurogenesis, angiogenesis, synaptogenesis, and antifibrosis of hepatocyte growth factor in rats brain after transient middle cerebral artery occlusion, J Neurosci Res, 2011;89:86-95

56. Sugiyama $Y$, Yagita $Y$, Oyama N, et al., Granulocyte colony-stimulating factor enhances arteriogenesis and ameliorates cerebral damage in a mouse model of ischemic stroke, stroke, 2011;42:770-5.

57. Murata Y, Rosell A, Scannevin RH, et al., Extension of the thrombolytic time window with minocycline in experimental stroke, stroke, 2008:39:3372-7.

58. Fagan SC, Waller JL, Nichols FT, et al., Minocycline to improve neurologic outcome in stroke (minos): A dose-finding study, Stroke, 2010;41:2283-7.

59. Navarro-Sobrino M, Rosell A, Penalba A, et al., Role of endogenous granulocyte-macrophage colony stimulating factor following stroke and relationship to neurological outcome, Curr Neurovasc Res, 2009;6:246-51.

60. Zhang $R$, Wang $L$, Zhang $L$, et al., Nitric oxide enhances angiogenesis via the synthesis of vascular endothelial growth factor and cgmp after stroke in the rat, Circ Res, 2003:92:308-13.

61. Cui X, Chopp M, Zacharek A, et al., Role of endothelial nitric oxide synthetase in arteriogenesis after stroke in mice, Neuroscience, 2009;159:744-50

62. Zhang $R$, Wang $Y$, Zhang L, et al., Sildenafil (viagra) induces neurogenesis and promotes functional recovery after stroke in rats, Stroke, 2002;33:2675-80.

63. Zhang RL, Zhang Z, Zhang L, et al., Delayed treatment with sildenafil enhances neurogenesis and improves functional recovery in aged rats after focal cerebral ischemia, J Neurosci Res, 2006;83:1213-9.

64. Chen J, Zhang ZG, Li Y, et al., Statins induce angiogenesis, neurogenesis, and synaptogenesis after stroke, Ann Neurol, 2003;53:743-51 
65. Zhang L, Zhang ZG, Ding GL, et al., Multitargeted effects of statin-enhanced thrombolytic therapy for stroke with recombinant human tissue-type plasminogen activator in the rat, Circulation, 2005;112:3486-94

66. Ding $G$, Jiang $Q$, Li L, et al., Mri of combination treatment of embolic stroke in rat with rtpa and atorvastatin, J Neurol Sci, 2006:246(1-2):139-47.

67. Morris DC, Chopp M, Zhang L, et al., Thymosin beta4 improves functional neurological outcome in a rat model of embolic stroke, Neuroscience, 2010;169:674-82.

68. Wang L, Zhang Z, Wang Y, et al., Treatment of stroke with erythropoietin enhances neurogenesis and angiogenesis and improves neurological function in rats, stroke, 2004;35:1732-7.

69. Li L, Jiang $Q$, Ding $G$, et al., Mri identification of white matter reorganization enhanced by erythropoietin treatment in a rat model of focal ischemia, Stroke, 2009:40:936-41.

70. Wang Y, Zhang ZG, Rhodes K, et al., Post-ischemic treatment with erythropoietin or carbamylated erythropoietin reduces infarction and improves neurological outcome in a rat mode of focal cerebral ischemia, Br J Pharmacol, 2007; 151(8):1377-84

71. Xiong Y, Mahmood A, Chopp M, Angiogenesis, neurogenesis and brain recovery of function following injury, Curr Opin Investig Drugs, 2010;11:298-308.
72. Zhang C, Chopp M, Cui Y, et al., Cerebrolysin enhances neurogenesis in the ischemic brain and improves functiona outcome after stroke, I Neurosci Res, 2010;88:3275-81.

73. Hong Z, Moessler $\mathrm{H}$, Bornstein $\mathrm{N}$, et al., A double-blind, placebo-controlled, randomized trial to evaluate the safety and efficacy of cerebrolysin in patients with acute ischacmic stroke in asia--casta, Int I Stroke, 2009;4:406-12.

74. Katakowski M, Zhang ZG, Chen J, et al., Phosphoinositide 3 kinase promotes adult subventricular neuroblast migration after stroke, J Neurosci Res, 2003;74:494-501.

75. Wang L, Chopp M, Gregg SR, et al., Neural progenitor cells treated with epo induce angiogenesis through the production of vegf, I Cereb Blood F Metab, 2008;28:1361-8.

76. Zhang L, Zhang ZG, Liu XS, et al., The pi3k/akt pathway mediates the neuroprotective effect of atorvastatin in extending thrombolytic therapy after embolic stroke in the rat, Arterioscler Thromb Vasc Biol, 2007:27:2470-5.

77. Wang L, Zhang ZG, Gregg SR, et al., The sonic hedgehog pathway mediates carbamylated erythropoietin-enhanced proliferation and differentiation of adult neural progenitor cells, I Biol Chem, 2007;282:32462-70.

78. Zhang J, Chen J, Li Y, et al., Niaspan treatment improves neurological functional recovery in experimental autoimmune encephalomyelitis mice, Neurobiol Dis, $2008 \cdot 32 \cdot 273-80$
79. Ehrenreich $\mathrm{H}$, Weissenborn $\mathrm{K}$, Prange $\mathrm{H}$, et al., Recombinant human erythropoietin in the treatment of acute ischemic stroke, Stroke, 2009;40:e647-56.

80. Jia L, Chopp M, Zhang L, et al., Erythropoietin in combination of tissue plasminogen activator exacerbates brain hemorrhage when treatment is initiated 6 hours after stroke, Stroke, 2010;41:2071-6.

81. Zhang R, Zhang L, Zhang Z, et al., A nitric oxide dono induces neurogenesis and reduces functional deficits after stroke in rats, Ann Neurol, 2001;50:602-11.

82. Zhang L, Zhang Z, Zhang RL, et al., Tadalafil, a long-acting type 5 phosphodiesterase isoenzyme inhibitor, improves neurological functional recovery in a rat model of embolic stroke, Brain research, 2006;1118:192-8.

83. Zhang $\mathrm{L}$, Zhang RL, Wang $\mathrm{Y}$, et al., Functional recovery in aged and young rats after embolic stroke: Treatment with a phosphodiesterase type 5 inhibitor, Stroke, 2005;36:847-52

84. Silver B, Grover KM, Arcila X, et al., Recovery in a patient with locked-in syndrome, Can I Neurol Sci, 2006;33:246-9.

85. Hanson LR, Liu XF Ross TM et al., Cerebrolysin Reduces Infarct Volume in a Rat Model of Focal Cerebral Ischemic Damage, Am J Neuroprot and Neuroreg, 2009;1:62-8.

86. Ladurner $G$, Kalvach P, Moessler H, Neuroprotective Treatment with Cerebrolysin in Patients with Acute Stroke: randomised controlled trial, INeural Transm, 2005:112:415-28. 